\title{
Search for an invisibly-decaying Higgs boson at LEP
}

\section{L3 Collaboration}

P. Achard ${ }^{\text {t }}$, O. Adriani ${ }^{\mathrm{q}}$, M. Aguilar-Benitez ${ }^{\mathrm{y}}$, J. Alcaraz ${ }^{\mathrm{y}}$, G. Alemanni ${ }^{\mathrm{w}}$, J. Allaby $^{\mathrm{r}}$, A. Aloisio ${ }^{\text {ac }}$, M.G. Alviggi ac, H. Anderhub ${ }^{\text {aw }}$, V.P. Andreev ${ }^{\text {f,ah }}$, F. Anselmo ${ }^{\text {h }}$,

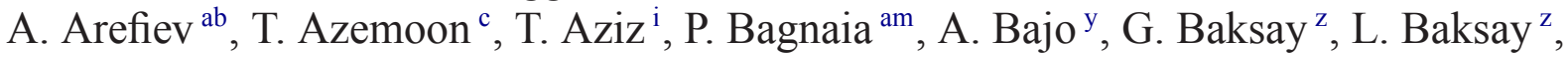
S.V. Baldew ${ }^{\text {b }}$, S. Banerjee ${ }^{\text {i, Sw. Banerjee }}{ }^{\text {d }}$, A. Barczyk ${ }^{\text {aw }, \text { au }}$, R. Barillère ${ }^{\text {r, }}$, P. Bartalini ${ }^{\mathrm{w}}$, M. Basile ${ }^{\mathrm{h}}$, N. Batalova ${ }^{\text {at }}$, R. Battiston ${ }^{\text {ag }}$, A. Bay ${ }^{\mathrm{w}}$, F. Becattini ${ }^{\mathrm{q}}$, U. Becker ${ }^{\mathrm{m}}$, F. Behner ${ }^{\text {aw }}$, L. Bellucci ${ }^{\mathrm{q}}$, R. Berbeco ${ }^{\mathrm{c}}$, J. Berdugo ${ }^{\mathrm{y}}$, P. Berges ${ }^{\mathrm{m}}$, B. Bertucci ${ }^{\text {ag }}$, B.L. Betev ${ }^{\text {aw }}$, M. Biasini ${ }^{\text {ag }}$, M. Biglietti ${ }^{\text {ac }}$, A. Biland ${ }^{\text {aw }}$, J.J. Blaising ${ }^{d}$, S.C. Blyth $^{\text {ai }}$, G.J. Bobbink ${ }^{\text {b }}$, A. Böhm ${ }^{\text {a }}$, L. Boldizsar ${ }^{1}$, B. Borgia ${ }^{\text {am }}$, S. Bottai ${ }^{\text {, }}$, D. Bourilkov ${ }^{\text {aw }}$, M. Bourquin ${ }^{\mathrm{t}}$, S. Braccini ${ }^{\mathrm{t}}$, J.G. Branson ${ }^{\text {ao }}$, F. Brochu ${ }^{\mathrm{d}}$, J.D. Burger ${ }^{\mathrm{m}}$, W.J. Burger ${ }^{\text {ag }}$, X.D. Cai ${ }^{\mathrm{m}}$, M. Capell ${ }^{\mathrm{m}}$, G. Cara Romeo ${ }^{\mathrm{h}}$, G. Carlino ${ }^{\text {ac }}$, A. Cartacci ${ }^{\mathrm{q}}$, J. Casaus ${ }^{\mathrm{y}}$, F. Cavallari am ${ }^{\mathrm{a}}$, N. Cavallo ${ }^{\text {aj }}$, C. Cecchi ${ }^{\text {ag }}$, M. Cerrada ${ }^{\mathrm{y}}$, M. Chamizo $^{\mathrm{t}}$, Y.H. Chang ar ${ }^{\text {a }}$ M. Chemarin ${ }^{\mathrm{x}}$, A. Chen ${ }^{\text {ar }}$, G. Chen ${ }^{\mathrm{g}}$, G.M. Chen ${ }^{\mathrm{g}}$, H.F. Chen ${ }^{\mathrm{v}}$, H.S. Chen ${ }^{\mathrm{g}}$, G. Chiefari ${ }^{\text {ac }}$, L. Cifarelli ${ }^{\text {an }}$, F. Cindolo ${ }^{\text {h }}$, I. Clare ${ }^{\mathrm{m}}$, R. Clare ${ }^{\mathrm{al}}$, G. Coignet ${ }^{\mathrm{d}}$, N. Colino ${ }^{\mathrm{y}}, \mathrm{S}$. Costantini ${ }^{\text {am }}$, B. de la Cruz ${ }^{\mathrm{y}}$, S. Cucciarelli ${ }^{\mathrm{ag}}$, R. de Asmundis ${ }^{\text {ac }}$, P. Déglon ${ }^{t}, J$. Debreczeni ${ }^{1}$, A. Degré ${ }^{\mathrm{d}}$, K. Dehmelt ${ }^{\mathrm{z}}, \mathrm{K}$. Deiters ${ }^{\text {au }}$, D. della Volpe ${ }^{\text {ac }}$, E. Delmeire ${ }^{t}$, P. Denes ${ }^{\text {ak }}$, F. DeNotaristefani ${ }^{\text {am }}$, A. De Salvo ${ }^{\text {aw }}$, M. Diemoz ${ }^{\text {am }}$, M. Dierckxsens ${ }^{\text {b }}$, C. Dionisi ${ }^{\text {am }}$, M. Dittmar ${ }^{\text {aw }}$, A. Doria ${ }^{\text {ac }}$, M.T. Dova ${ }^{\mathrm{j}, 5}$, D. Duchesneau ${ }^{d}$, M. Duda ${ }^{a}$, B. Echenard ${ }^{t}$, A. Eline ${ }^{\mathrm{r}}$, A. El Hage ${ }^{\mathrm{a}}$, H. El Mamouni ${ }^{\mathrm{x}}$, A. Engler ${ }^{\text {ai }}$, F.J. Eppling ${ }^{\mathrm{m}}$, P. Extermann ${ }^{\mathrm{t}}$, M.A. Falagan ${ }^{\mathrm{y}}$, S. Falciano ${ }^{\text {am }}$, A. Favara ${ }^{\text {af }}$, J. Fay ${ }^{x}$, O. Fedin ${ }^{\text {ah }}$, M. Felcini ${ }^{\text {aw }}$, T. Ferguson ${ }^{\text {ai }}$, H. Fesefeldt ${ }^{a}$, E. Fiandrini ${ }^{\text {ag }}$, J.H. Field ${ }^{\mathrm{t}}$, F. Filthaut ${ }^{\text {ae }}$, P.H. Fisher ${ }^{\mathrm{m}}$, W. Fisher ${ }^{\text {ak }}$, I. Fisk ${ }^{\text {ao }}$, G. Forconi ${ }^{\mathrm{m}}$, K. Freudenreich ${ }^{\text {aw }}$, C. Furetta ${ }^{\text {aa }}$, Yu. Galaktionov ${ }^{\text {ab,m }}$, S.N. Ganguli ${ }^{i}$, P. Garcia-Abia ${ }^{y}$, M. Gataullin ${ }^{\text {af }}$, S. Gentile ${ }^{\text {am }}$, S. Giagu ${ }^{\text {am }}$, Z.F. Gong ${ }^{\text {v }}$, G. Grenier ${ }^{\mathrm{x}}$, O. Grimm ${ }^{\text {aw }}$, M.W. Gruenewald ${ }^{\mathrm{p}}$, M. Guida ${ }^{\text {an }}$, V.K. Gupta ${ }^{\mathrm{ak}}$, A. Gurtu ${ }^{\mathrm{i}}$, L.J. Gutay ${ }^{\text {at }}$, D. Haas ${ }^{\mathrm{e}}$,

D. Hatzifotiadou ${ }^{\text {h }}$, T. Hebbeker ${ }^{\mathrm{a}}$, A. Hervé ${ }^{\mathrm{r}}$, J. Hirschfelder ${ }^{\text {ai }}, \mathrm{H}$. Hofer ${ }^{\text {aw }}$, M. Hohlmann ${ }^{\text {z }}$, G. Holzner ${ }^{\text {aw }}$, S.R. Hou ${ }^{\text {ar }}$, B.N. Jin ${ }^{\text {g }}$, P. Jindal ${ }^{\text {n }}$, L.W. Jones ${ }^{\text {c }}$, P. de Jong ${ }^{\mathrm{b}}$, I. Josa-Mutuberría ${ }^{\mathrm{y}}$, M. Kaur ${ }^{\mathrm{n}}$, M.N. Kienzle-Focacci ${ }^{\mathrm{t}}$, J.K. Kim ${ }^{\text {aq }}$, 
J. Kirkby ${ }^{\text {r }}$ W. Kittel ${ }^{\text {ae }}$, A. Klimentov ${ }^{\text {m,ab }}$, A.C. König ${ }^{\text {ae }}$, M. Kopal ${ }^{\text {at }}$, V. Koutsenko ${ }^{\text {m,ab }}$, M. Kräber ${ }^{\text {aw }}$, R.W. Kraemer ${ }^{\text {ai }}$, A. Krüger ${ }^{\text {av }}$, A. Kunin ${ }^{\text {m }}$, P. Ladron de Guevara ${ }^{y}$, I. Laktineh ${ }^{\mathrm{x}}$, G. Landi ${ }^{\mathrm{q}}$, M. Lebeau ${ }^{\mathrm{r}}$, A. Lebedev ${ }^{\mathrm{m}}$, P. Lebrun ${ }^{\mathrm{x}}$, P. Lecomte ${ }^{\text {aw }}$, P. Lecoq ${ }^{r}$, P. Le Coultre ${ }^{\text {aw }}$, J.M. Le Goff ${ }^{\mathrm{r}}$, R. Leiste ${ }^{\text {av }}$, M. Levtchenko ${ }^{\text {aa }}$, P. Levtchenko ah ${ }^{\text {a }}$ C. Li $^{\text {v }}$, S. Likhoded ${ }^{\text {av }}$, C.H. Lin ${ }^{\text {ar }}$, W.T. Lin ${ }^{\text {ar }}$, F.L. Linde ${ }^{\text {b }}$, L. Lista ${ }^{\text {ac }}$, Z.A. Liu ${ }^{\mathrm{g}}$, W. Lohmann ${ }^{\text {av }}$, E. Longo ${ }^{\text {am }}$, Y.S. Lu ${ }^{\mathrm{g}}$, C. Luci $^{\mathrm{am}}$, L. Luminari ${ }^{\text {am }}$, W. Lustermann ${ }^{\text {aw }}$, W.G. Ma ${ }^{\mathrm{v}}$, L. Malgeri ${ }^{\mathrm{r}}$, A. Malinin ${ }^{\mathrm{ab}}$, C. Maña ${ }^{\mathrm{y}}$, J. Mans ${ }^{\mathrm{ak}}$, J.P. Martin ${ }^{\mathrm{x}}$, F. Marzano ${ }^{\text {am }}$, K. Mazumdar ${ }^{\mathrm{i}}$, R.R. McNeil ${ }^{\mathrm{f}}$, S. Mele ${ }^{\mathrm{r}, \mathrm{ac}}$, L. Merola ${ }^{\text {ac }}$, M. Meschini ${ }^{\mathrm{q}}$, W.J. Metzger ${ }^{\text {ae }}$, A. Mihul ${ }^{\mathrm{k}}$, H. Milcent ${ }^{\mathrm{r}}$, G. Mirabelli ${ }^{\text {am }}$, J. Mnich ${ }^{\mathrm{a}}$, G.B. Mohanty ${ }^{\mathrm{i}}$, G.S. Muanza ${ }^{\mathrm{x}}$, A.J.M. Muijs ${ }^{\mathrm{b}}$, B. Musicar ${ }^{\mathrm{ao}}$, M. Musy ${ }^{\mathrm{am}}$, S. Nagy ${ }^{\mathrm{o}}$, S. Natale ${ }^{\mathrm{t}}$, M. Napolitano ${ }^{\text {ac }}$, F. Nessi-Tedaldi aw , H. Newman ${ }^{\text {af }}$, A. Nisati ${ }^{\text {am }}$, T. Novak ${ }^{\text {ae }}$, H. Nowak ${ }^{\text {av }}$, R. Ofierzynski ${ }^{\text {aw }}$, G. Organtini ${ }^{\text {am }}$, I. Pal $^{\text {at }}$, C. Palomares ${ }^{\mathrm{y}}$, P. Paolucci ${ }^{\text {ac }}$,

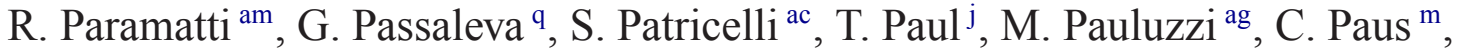
F. Pauss ${ }^{\text {aw }}$, M. Pedace ${ }^{\text {am }}$, S. Pensotti aa ${ }^{\text {a }}$ D. Perret-Gallix ${ }^{\mathrm{d}}$, D. Piccolo ${ }^{\text {ac }}$, F. Pierella ${ }^{\mathrm{h}}$, M. Pioppi ${ }^{\text {ag }}$, P.A. Piroué ${ }^{a k}$, E. Pistolesi ${ }^{\text {aa }}$, V. Plyaskin ${ }^{\text {ab }}$, M. Pohl ${ }^{t}$, V. Pojidaev ${ }^{\mathrm{q}}$, J. Pothier ${ }^{\mathrm{r}}$, D. Prokofiev ${ }^{\text {ah }}$, J. Quartieri ${ }^{\text {an }}$, G. Rahal-Callot ${ }^{\text {aw }}$, M.A. Rahaman ${ }^{\mathrm{i}}$, P. Raics ${ }^{\circ}$, N. Raja ${ }^{i}$, R. Ramelli ${ }^{\text {aw }}$, P.G. Rancoita ${ }^{\text {aa }}$, R. Ranieri ${ }^{\text {q }}$, A. Raspereza ${ }^{\text {av }}$, P. Razis ${ }^{\text {ad }}$, D. Ren ${ }^{\text {aw }}$, M. Rescigno ${ }^{\text {am }}$, S. Reucroft ${ }^{\mathrm{j}}$, S. Riemann $^{\text {av }}$, K. Riles ${ }^{\text {c }}$, B.P. Roe ${ }^{c}$, L. Romero ${ }^{y}$, A. Rosca ${ }^{\text {av }}$, C. Rosemann ${ }^{\text {a }}$, C. Rosenbleck ${ }^{a}$, S. Rosier-Lees ${ }^{d}$, S. Roth ${ }^{\text {a }}$, J.A. Rubio ${ }^{\text {r }}$, G. Ruggiero ${ }^{\text {q }}$, H. Rykaczewski ${ }^{\text {aw }}$, A. Sakharov ${ }^{\text {aw }}$, S. Saremi ${ }^{\text {f }}$, S. Sarkar ${ }^{\text {am }}$, J. Salicio ${ }^{r}$, E. Sanchez ${ }^{y}$, C. Schäfer ${ }^{r}$, V. Schegelsky ${ }^{\text {ah }}$, H. Schopper ${ }^{\text {u }}$, D.J. Schotanus ${ }^{\text {ae }}$, C. Sciacca ${ }^{\text {ac }}$, L. Servoli ${ }^{\text {ag }}$, S. Shevchenko af ${ }^{\text {af }}$ N. Shivarov ap, V. Shoutko ${ }^{m}$, E. Shumilov ${ }^{\text {ab }}$, A. Shvorob ${ }^{\text {af }}$, D. Son ${ }^{\text {aq }}$, C. Souga ${ }^{\mathrm{x}}$, P. Spillantini ${ }^{\mathrm{q}}$, M. Steuer ${ }^{\mathrm{m}}$, D.P. Stickland ${ }^{\text {ak }}$, B. Stoyanov ap, A. Straessner ${ }^{\mathrm{t}}$, K. Sudhakar ${ }^{\mathrm{i}}$, G. Sultanov ${ }^{\text {ap }}$, L.Z. Sun ${ }^{\text {v }, ~ S . ~ S u s h k o v ~}{ }^{\mathrm{a}}$, H. Suter ${ }^{\text {aw }}$, J.D. Swain ${ }^{\mathrm{j}}$, Z. Szillasi ${ }^{\mathrm{z}, 3}$, X.W. Tang ${ }^{\mathrm{g}}$, P. Tarjan ${ }^{\mathrm{o}}$, L. Tauscher ${ }^{\mathrm{e}}, \mathrm{L}_{\text {. Taylor }}^{\mathrm{j}}$, B. Tellili ${ }^{\mathrm{x}}, \mathrm{D}$. Teyssier ${ }^{\mathrm{x}}$, C. Timmermans ${ }^{\text {ae }}$, Samuel C.C. Ting ${ }^{\mathrm{m}}$, S.M. Ting ${ }^{\mathrm{m}}$, S.C. Tonwar ${ }^{\mathrm{i}}$, J. Tóth ${ }^{1}$, C. Tully ${ }^{\mathrm{ak}}$, K.L. Tung ${ }^{\mathrm{g}}$, J. Ulbricht ${ }^{\text {aw }}$, E. Valente ${ }^{\text {am }}$, R.T. Van de Walle ${ }^{\text {ae }}$, R. Vasquez ${ }^{\text {at }}$, V. Veszpremi ${ }^{\mathrm{z}}$, G. Vesztergombi ${ }^{1}$, I. Vetlitsky ${ }^{\mathrm{ab}}$, D. Vicinanza ${ }^{\text {an }}$, G. Viertel $^{\text {aw }}$, S. Villa ${ }^{\text {al }}$, M. Vivargent ${ }^{\text {d }}$, S. Vlachos ${ }^{\text {e }}$, I. Vodopianov ${ }^{z}, H$. Vogel ${ }^{\text {ai }}$, H. Vogt ${ }^{\text {av }}$, I. Vorobiev ${ }^{\text {ai,ab }}$, A.A. Vorobyov ${ }^{\text {ah }}$, M. Wadhwa ${ }^{\mathrm{e}}$, Q. Wang ${ }^{\text {ae }}$, X.L. Wang ${ }^{\mathrm{v}}$, Z.M. Wang ${ }^{\mathrm{v}}$, M. Weber ${ }^{\mathrm{r}}$, S. Wynhoff ${ }^{\text {ak }}$, L. Xia ${ }^{\text {af }}$, Z.Z. Xu ${ }^{v}$, J. Yamamoto ${ }^{\text {c }}$, B.Z. Yang ${ }^{v}$, C.G. Yang ${ }^{g}$, H.J. Yang ${ }^{\text {, }}$, M. Yang ${ }^{g}$, S.C. Yeh ${ }^{\text {as }}$, An. Zalite ${ }^{\text {ah }}$, Yu. Zalite ${ }^{\text {ah }}$, Z.P. Zhang ${ }^{v}$, J. Zhao $^{\text {v }}$, G.Y. Zhu ${ }^{\mathrm{g}}$,

R.Y. Zhu ${ }^{\text {af }}$, H.L. Zhuang ${ }^{\text {g }}$, A. Zichichi ${ }^{\text {h,r,s }}$, B. Zimmermann ${ }^{\text {aw }}$, M. Zöller ${ }^{\text {a }}$

${ }^{a}$ III. Physikalisches Institut, RWTH, D-52056 Aachen, Germany ${ }^{1}$

${ }^{\mathrm{b}}$ National Institute for High Energy Physics, NIKHEF, and University of Amsterdam, NL-1009 DB Amsterdam, The Netherlands c University of Michigan, Ann Arbor, MI 48109, USA

d Laboratoire d'Annecy-le-Vieux de Physique des Particules, LAPP, IN2P3-CNRS, BP 110, F-74941 Annecy-le-Vieux cedex, France e Institute of Physics, University of Basel, CH-4056 Basel, Switzerland 


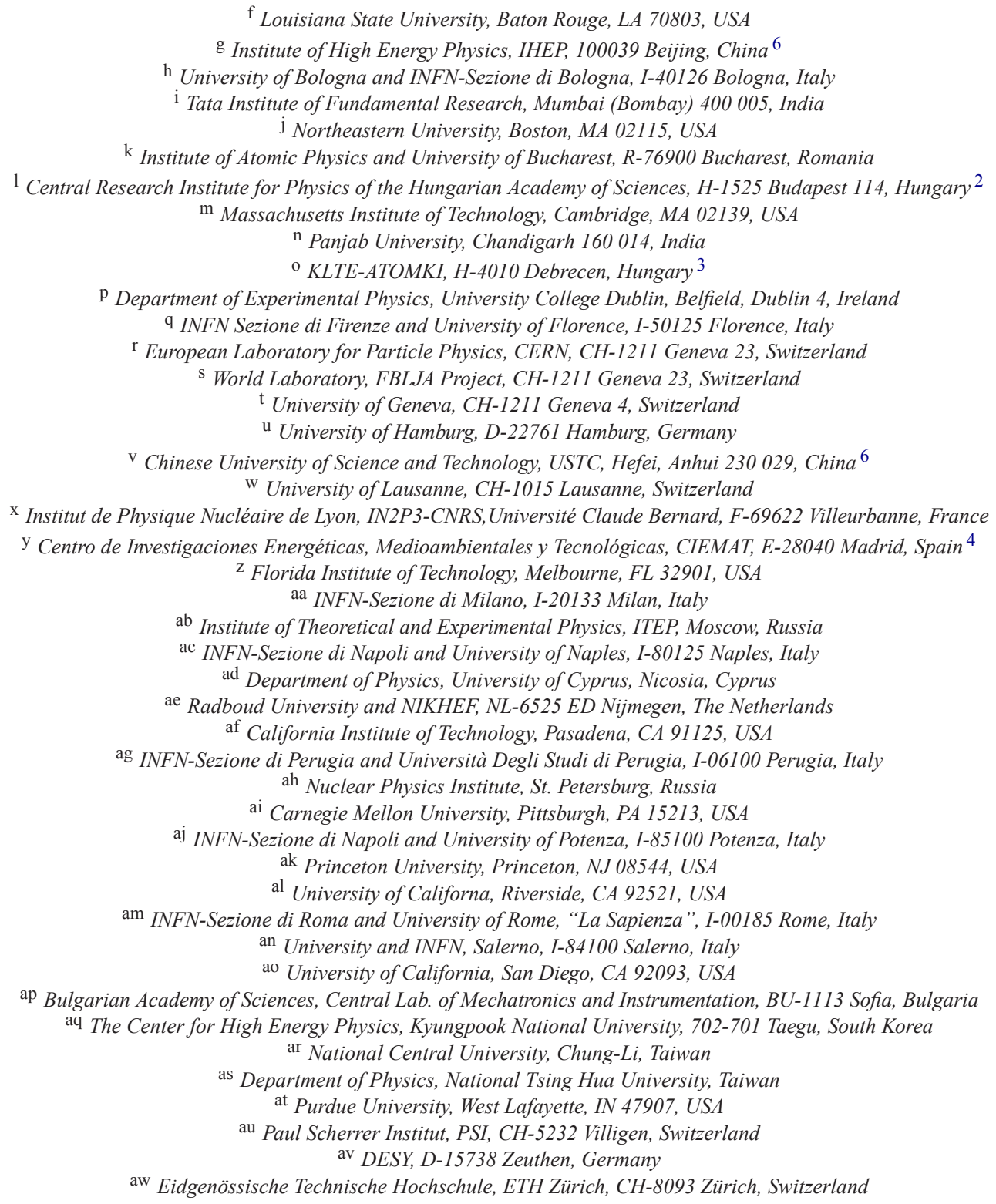

Received 1 November 2004; accepted 13 January 2005

Available online 20 January 2005

Editor: L. Rolandi

${ }^{1}$ Supported by the German Bundesministerium für Bildung, Wissenschaft, Forschung und Technologie.

2 Supported by the Hungarian OTKA fund under contract numbers T019181, F023259 and T037350.

3 Also supported by the Hungarian OTKA fund under contract number T026178.

4 Supported also by the Comisión Interministerial de Ciencia y Tecnología.

5 Also supported by CONICET and Universidad Nacional de La Plata, CC 67, 1900 La Plata, Argentina.

6 Supported by the National Natural Science Foundation of China. 


\begin{abstract}
A search for a Higgs boson produced in $\mathrm{e}^{+} \mathrm{e}^{-}$collisions in association with a $\mathrm{Z}$ boson and decaying into invisible particles is performed. Data collected at LEP with the L3 detector at centre-of-mass energies from 189 to $209 \mathrm{GeV}$ are used, corresponding to an integrated luminosity of $0.63 \mathrm{fb}^{-1}$. Events with hadrons, electrons or muons with visible masses compatible with a $\mathrm{Z}$ boson and missing energy and momentum are selected. They are consistent with the Standard Model expectations. A lower limit of $112.3 \mathrm{GeV}$ is set at $95 \%$ confidence level on the mass of the invisibly-decaying Higgs boson in the hypothesis that its production cross section equals that of the Standard Model Higgs boson. Relaxing this hypothesis, upper limits on the production cross section are derived.
\end{abstract}

(C) 2005 Published by Elsevier B.V.

\section{Introduction}

The Standard Model of the electroweak interactions [1] relies on the Higgs mechanism [2] to explain the observed masses of the elementary particles. A consequence of this mechanism is the existence of an additional particle, the Higgs boson. Direct searches at the LEP $\mathrm{e}^{+} \mathrm{e}^{-}$collider for the Standard Model Higgs boson, $\mathrm{H}$, produced in the Higgs-strahlung process $\mathrm{e}^{+} \mathrm{e}^{-} \rightarrow \mathrm{HZ}$ did not observe a significant excess of events over the Standard Model expectations [3-5]. These searches are based on the hypothesis that the Higgs boson mainly decays into b quarks. Searches in which this hypothesis is relaxed and the Higgs boson is allowed to decay into a generic hadronic final state also yield negative results [6]. In addition, no signs of the Higgs boson were found in cases in which anomalous couplings would affect its production and decay mechanisms [7].

However, a Higgs boson which decays into stable weakly-interacting particles would have escaped detection in all these searches. Such possibility has been extensively proposed in literature for the case of Higgs bosons decaying into the lightest supersymmetric particles [8], fourth-generation neutrinos [9], neutrinos in the context of theories with extra space dimensions [10], majorons $[10,11]$ or into a general scalar gauge singlet added to the Standard Model [12].

This Letter describes the search for an invisiblydecaying Higgs boson, h, produced through the Higgsstrahlung process $\mathrm{e}^{+} \mathrm{e}^{-} \rightarrow \mathrm{hZ}$. Decays of the $\mathrm{Z}$ boson into hadrons, electron pairs and muon pairs are considered and analyses are devised to select events with hadrons or leptons and missing energy and momentum. Data collected by the L3 detector [13] at LEP at centre-of-mass energies $\sqrt{s}=189-209 \mathrm{GeV}$ are analysed. They correspond to a total integrated luminosity of $0.63 \mathrm{fb}^{-1}$, as detailed in Table 1 .

The results presented in this Letter supersede those of previous L3 studies [14,15], as the complete highluminosity and high-energy data sample is investigated and the previously published data collected at $\sqrt{s}=189 \mathrm{GeV}$ [15] are re-analysed with improved procedures. Similar searches were also performed by other LEP Collaborations $[3,16]$.

\section{Event simulation}

To optimise the selection criteria and determine the efficiency to detect a possible signal, samples of Higgs-boson events are generated using the PYTHIA Monte Carlo program [17] for masses of the Higgs boson, $m_{\mathrm{h}}$, between 50 and $120 \mathrm{GeV}$ in steps between 5 and $10 \mathrm{GeV}$.

The following Monte Carlo programs are used to model Standard Model processes: KK2f [18] for $\mathrm{e}^{+} \mathrm{e}^{-} \rightarrow \mathrm{q} \overline{\mathrm{q}}, \mathrm{e}^{+} \mathrm{e}^{-} \rightarrow \mu^{+} \mu^{-}$and $\mathrm{e}^{+} \mathrm{e}^{-} \rightarrow \tau^{+} \tau^{-}, \mathrm{BH}-$ WIDE [19] for Bhabha scattering, and PHOJET [20] and DIAG36 [21] for hadron and lepton production in two-photon collisions, respectively. Four-fermion final states relevant for the analysis of events with hadrons

Table 1

Centre-of-mass energies and corresponding integrated luminosities, $\mathcal{L}$, considered in this analysis

\begin{tabular}{|c|c|c|c|c|c|c|c|c|}
\hline$\sqrt{s}[\mathrm{GeV}]$ & 188.6 & 191.6 & 195.6 & 199.5 & 201.7 & $202.5-205.5$ & $205.5-207.5$ & $207.5-209.2$ \\
\hline $\mathcal{L}\left[\mathrm{pb}^{-1}\right]$ & 176.8 & 29.7 & 83.9 & 82.8 & 39.1 & 77.8 & 131.4 & 8.2 \\
\hline
\end{tabular}


Table 2

Results of the selection of events with hadrons and missing energy. The lower part of the table details the composition of the expected Standard Model sample. The uncertainties reflect the limited background Monte Carlo statistics

\begin{tabular}{llll}
\hline & Preselection & Light-Higgs selection & Heavy-Higgs selection \\
\hline Data & 779 & 345 & 130 \\
Standard Model & $771.8 \pm 3.6$ & $347.2 \pm 2.0$ & $127.1 \pm 1.8$ \\
Two-photon interactions & $6.4 \pm 1.6$ & - & $2.7 \pm 1.1$ \\
Two-fermion final states & $69.9 \pm 1.6$ & $2.6 \pm 0.3$ & $21.4 \pm 0.8$ \\
Four-fermion final states & $695.5 \pm 2.8$ & $344.6 \pm 2.0$ & $103.0 \pm 1.1$ \\
\hline
\end{tabular}

and missing energy are generated with PYTHIA for Z-boson pair-production and the $\mathrm{e}^{+} \mathrm{e}^{-} \rightarrow \mathrm{Ze}^{+} \mathrm{e}^{-}$ process and with KORALW [22] for W-boson pairproduction, with the exception of the $\mathrm{e}^{+} \mathrm{e}^{-} \rightarrow \mathrm{Wev} \rightarrow$ qqev process, modelled with EXCALIBUR [23]. All four-fermion processes with charged leptons and neutrinos in the final states, relevant for the analysis of events with leptons and missing energy, are generated with KandY [24].

For each centre-of-mass energy, the number of simulated background events corresponds to at least 50 times the number of expected events, up to a maximum of 7.5 million KandY events, except for twophoton interactions and Bhabha scattering for which twice and seven times the collected luminosity is simulated, respectively.

The L3 detector response is simulated using the GEANT program [25], which takes into account the effects of energy loss, multiple scattering and showering in the detector. Time-dependent inefficiencies of the different subdetectors, as monitored during the data-taking period, are taken into account in the simulation procedure.

\section{Selection of events with hadrons and missing energy}

A preselection identifies events compatible with the production of a heavy invisible particle and a $\mathrm{Z}$ boson decaying into hadrons. High multiplicity events are retained if their visible energy, $E_{\mathrm{vis}}$, satisfies $0.3<$ $E_{\text {vis }} / \sqrt{s}<0.65$ and have a visible mass between 60 and $115 \mathrm{GeV}$. No identified leptons or photons of energy above $10 \mathrm{GeV}$ are allowed in these events. To suppress the large background from hadron production in two-photon collisions and events from the $\mathrm{e}^{+} \mathrm{e}^{-} \rightarrow \mathrm{q} \overline{\mathrm{q}} \gamma$ process with a high-energy and low polar-angle photon, the missing momentum of the event is required to point inside the detector: its polar angle with respect to the beam axis, $\theta_{\text {miss }}$, must satisfy $\left|\cos \theta_{\text {miss }}\right|<0.9$. In addition, the event is reconstructed into two jets by means of the DURHAM algorithm [26] and the angle between the jets is required to be smaller than $175^{\circ}$. Events with large energy deposits in the low-angle calorimeters are also rejected. After the preselection, 779 events are selected in data while 772 events are expected from Standard Model processes, as detailed in Table 2. The signal efficiencies depend on $m_{\mathrm{h}}$, and vary from 52 up to $59 \%$. Up to $90 \%$ of the background comes from fourfermion processes and $10 \%$ from fermion-pair production.

Two selections are devised in order to retain high efficiency for light and heavy Higgs bosons. The "light-Higgs selection" is applied to events where the relativistic velocity of the reconstructed hadron system, $\beta$, satisfies $\beta>0.4$. The "heavy-Higgs selection" is applied to the remaining events.

The dominant background for the light-Higgs selection arises from $\mathrm{W}$ boson pair-production where one of the $\mathrm{W}$ bosons decays into hadrons and the other into leptons and from the $\mathrm{e}^{+} \mathrm{e}^{-} \rightarrow$ Wev process. Two additional selection criteria are applied to reduce these backgrounds: $\zeta_{\text {jet }}<100^{\circ}$ and $\theta_{3}<330^{\circ}$, where $\zeta_{\text {jet }}$ is the angle between the jets in the plane transverse to the beam direction and $\theta_{3}$ is the sum of the three inter-jet angles defined if the event is reconstructed into a threejet topology with the DURHAM algorithm. The last cut rejects genuine three-jet events from W-boson pairproduction where a W-boson decays into hadrons and the other into tau leptons which decay into hadrons. Fig. 1(a) and (b) present the distributions of $\zeta_{\text {jet }}$ and $\theta_{3}$.

The heavy-Higgs selection enforces the topology of a heavy undetected particle by means of two cuts against the background from pair production of ei- 

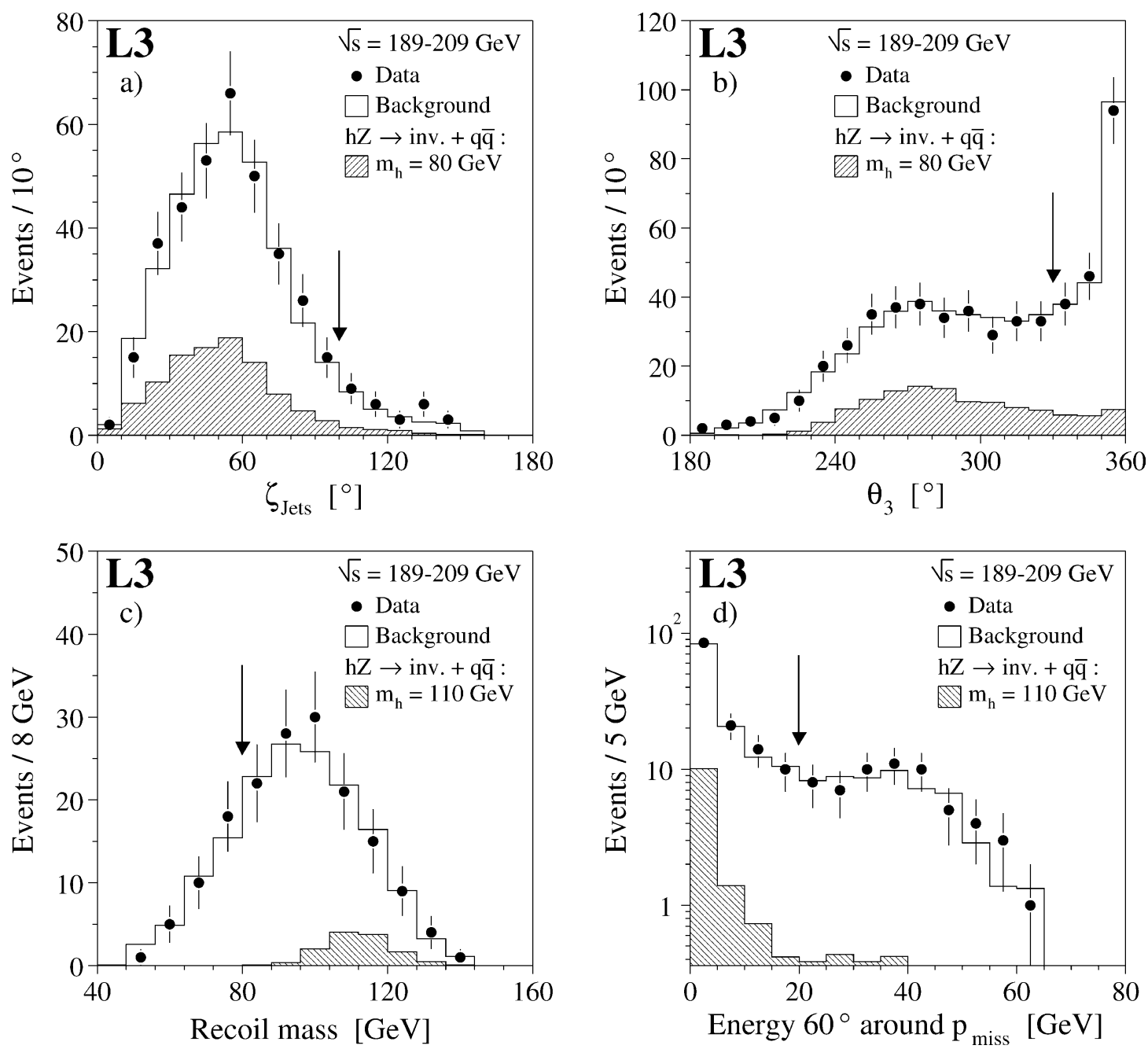

Fig. 1. Distributions of variables used in the selection of events with hadrons and missing energy. The dots represent the data, the open area the sum of all background contributions and the hatched histogram the expectation for a signal. The arrows represent the position of the selection criteria.

ther $\mathrm{W}$ bosons or fermions. The mass recoiling to the hadron system is required to be greater than $80 \mathrm{GeV}$ and the energy deposited in the calorimeters in a $60^{\circ}$ cone around the direction of the missing momentum is required to be smaller than $20 \mathrm{GeV}$. The distributions of these variables are shown in Fig. 1(c) and (d).

The results of the light- and heavy-Higgs selections are presented in Table 2, while Table 3 lists the signal efficiencies. In total, 475 events are selected in data and 474 are expected from Standard Model processes, dominated by four fermion
Table 3

Selection efficiencies as a function of the mass of the invisiblydecaying Higgs boson. The uncertainties are due to the limited signal Monte Carlo statistics

\begin{tabular}{llll}
\hline \multicolumn{4}{l}{ Efficiency [\%] } \\
\hline$m_{\mathrm{h}}(\mathrm{GeV})$ & $\mathrm{Z} \rightarrow \mathrm{q} \overline{\mathrm{q}}$ & $\mathrm{Z} \rightarrow \mathrm{e}^{+} \mathrm{e}^{-}$ & $\mathrm{Z} \rightarrow \mu^{+} \mu^{-}$ \\
\hline 60 & $49.0 \pm 1.5$ & $34.2 \pm 0.9$ & $22.4 \pm 0.8$ \\
70 & $49.8 \pm 1.6$ & $38.0 \pm 0.8$ & $26.6 \pm 0.7$ \\
80 & $49.1 \pm 1.8$ & $44.9 \pm 0.8$ & $32.7 \pm 0.8$ \\
90 & $50.2 \pm 1.9$ & $49.9 \pm 0.8$ & $31.2 \pm 0.8$ \\
100 & $49.4 \pm 1.9$ & $40.1 \pm 0.8$ & $27.0 \pm 0.7$ \\
110 & $47.6 \pm 1.7$ & $23.3 \pm 0.8$ & $14.8 \pm 0.7$ \\
\hline
\end{tabular}



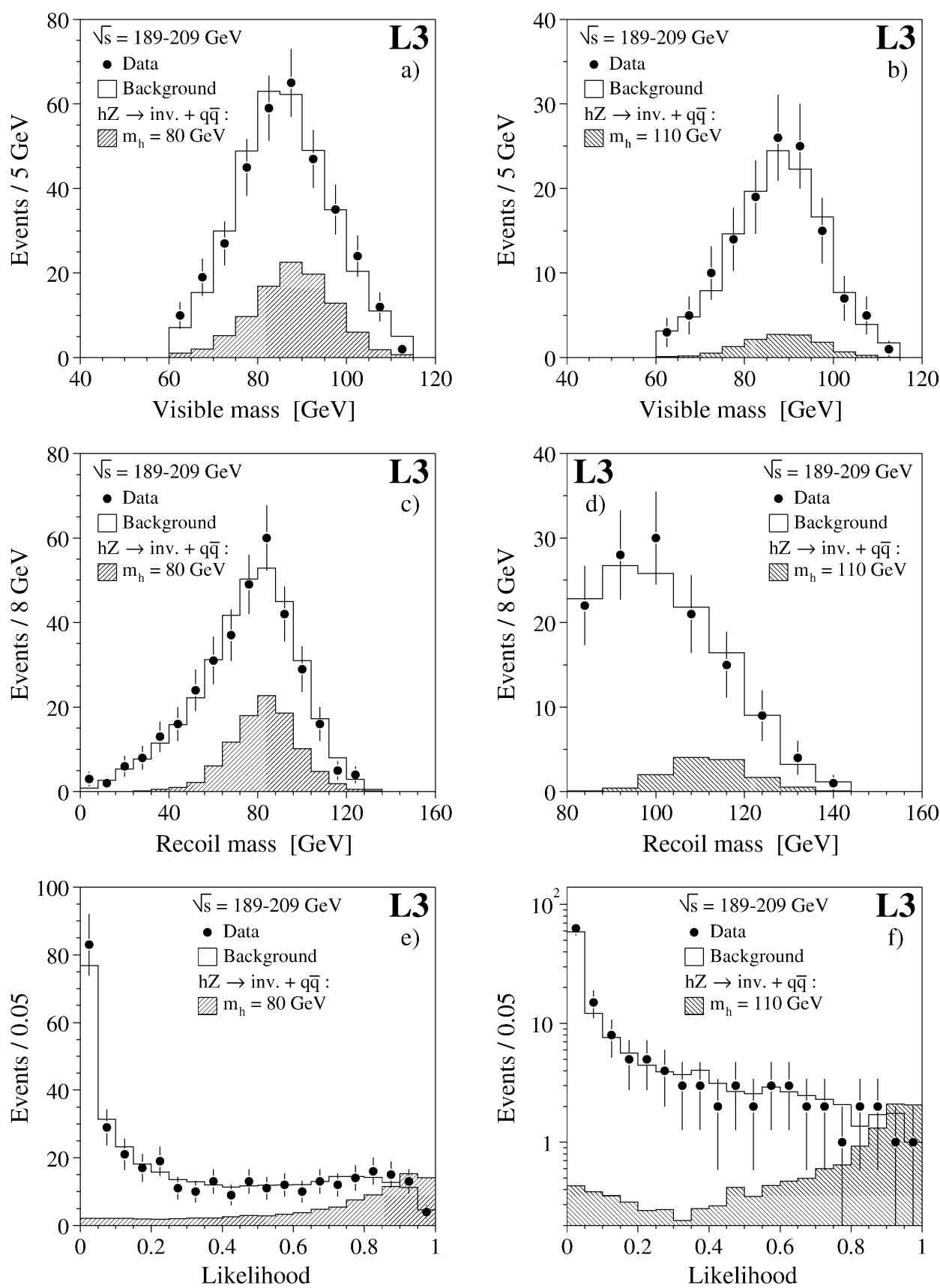

Fig. 2. Distributions of the visible and recoil masses for events selected by the light- and heavy-Higgs analyses of events with hadrons and missing energy. The dots represent the data, the open area the sum of all background contributions and the hatched histogram the expectation for a signal. The distributions of the final discriminants used in the analysis are also shown.

final-states. Fig. 2(a)-(d) present the distributions of the visible mass of the hadronic system and of the mass recoiling to the hadronic system, for the light- and heavy-Higgs selections. No indication for an excess of events in the signal regions is observed. 
The sensitivity to a possible Higgs signal is enhanced by building a discriminating variable for each of the two analyses. This variable combines [27] information from the visible and recoil masses, as well as the jet widths and the parameter $y_{23}$ of the DURHAM algorithm for which three jets are reconstructed in a two-jet event. Fig. 2(e) and (f) present the distributions of the discriminating variable for events selected by the light- and heavy-Higgs selections, respectively. A good agreement between the observations and the Standard Model predictions is observed.

\section{Selection of events with leptons and missing energy}

The selection of events possibly originating from an invisibly-decaying Higgs boson and a $\mathrm{Z}$ boson decaying into leptons proceeds from the L3 analysis of $\mathrm{W}$-boson pair-production where either both $\mathrm{W}$ bosons decay into an electron and a neutrino, or both decay into a muon and a neutrino [28]. Events with an electron or a muon pair are selected if the least and most energetic leptons have energies above 5 and $25 \mathrm{GeV}$, respectively. The angle of the leptons with respect to the beam direction, $\theta$, must satisfy $|\cos \theta|<0.96$. In the case of electrons, to reduce the background from the forward-peaked Bhabha scattering, at least one of the electrons must satisfy $|\cos \theta|<0.92$. To suppress background from fermion pair-production and cosmic rays, the angle between the two leptons in the plane transverse to the beam direction, $\zeta_{\ell}$, must satisfy $\zeta_{\ell}<172^{\circ}$. Residual background from cosmic rays is rejected by requiring the leptons to have a signal in the scintillator time-of-flight counters compatible with the beam crossing. Finally, the presence of undetected particles is enforced by requiring the event momentum transverse to the beam direction, $p_{t}$, to be greater than $8 \mathrm{GeV}$.

A total of 147 electron pairs and 115 muon pairs are selected, in good agreement with the Standard Model expectation of 136 and 130 events, respectively. These events are mostly due to four-fermion production, as summarised in Table 4. Signal efficiencies depend on $m_{\mathrm{h}}$ and are about 60 and $50 \%$ for final states with electrons and muons, respectively. The distributions after this preselection of the visible and recoil masses of the lepton pairs, as well as of the visible energy of the events are shown in Fig. 3. A good agreement between data and Monte Carlo expectations is found.

The main criteria to isolate signal events is to require the consistency of the visible mass with the mass of the $\mathrm{Z}$ boson. Two ranges are chosen, $86-95 \mathrm{GeV}$ for electrons and 80-99 GeV for muons, as illustrated in Fig. 3(a) and (b). In addition, the event selection requires $\left|\cos \theta_{\text {miss }}\right|<0.9$. In order to reduce the fourfermion background and increase the signal sensitivity, events are classified according to the value of the recoil mass. If it is below $85 \mathrm{GeV}$, a light-Higgs selection is further applied. A heavy-Higgs selection is applied otherwise. The light-Higgs selection relies on three cuts, common to both final states: $\zeta_{\ell}>100^{\circ}$, $E_{\mathrm{vis}} / \sqrt{s}<0.57$ and $p_{z} / \sqrt{s}<0.25$, where $p_{z}$ is the projection of the event momentum along the direction of the beams. In addition, events with muons are required to satisfy $p_{t}>14 \mathrm{GeV}$. The heavy-Higgs selection requires $E_{\text {vis }} / \sqrt{s}<0.45$ for both final states and $p_{t}>20 \mathrm{GeV}$ for final states with muons.

After these cuts, a total of 6 events are observed in the electron final-state and 9 in the muon final state,

Table 4

Results of the selection of events with leptons and missing energy. The lower part of the table details the composition of the expected Standard Model sample. The statistical uncertainties on the background estimation are negligible

\begin{tabular}{lllll}
\hline & $\mathrm{Z} \rightarrow \mathrm{e}^{+} \mathrm{e}^{-}$ & & $\mathrm{Z} \rightarrow \mu^{+} \mu^{-}$ \\
\cline { 2 - 4 } & Preselection & Selection & Preselection & 115 \\
\hline Data & 147 & 6.0 & 130.2 & 9 \\
Standard Model & 136.4 & 9.7 & - & 11.1 \\
$\mathrm{e}^{+} \mathrm{e}^{-} \rightarrow \mathrm{e}^{+} \mathrm{e}^{-}(\gamma)$ & 19.7 & 0.3 & 12.3 & - \\
$\mathrm{e}^{+} \mathrm{e}^{-} \rightarrow \mu^{+} \mu^{-}(\gamma)$ & - & - & 1.1 & 0.8 \\
$\mathrm{e}^{+} \mathrm{e}^{-} \rightarrow \tau^{+} \tau^{-}(\gamma)$ & 1.5 & - & 30.6 & - \\
Two-photon interactions & 6.9 & - & 86.2 & 10.3 \\
Four-fermion final states & 108.3 & 9.4 & & - \\
\hline
\end{tabular}



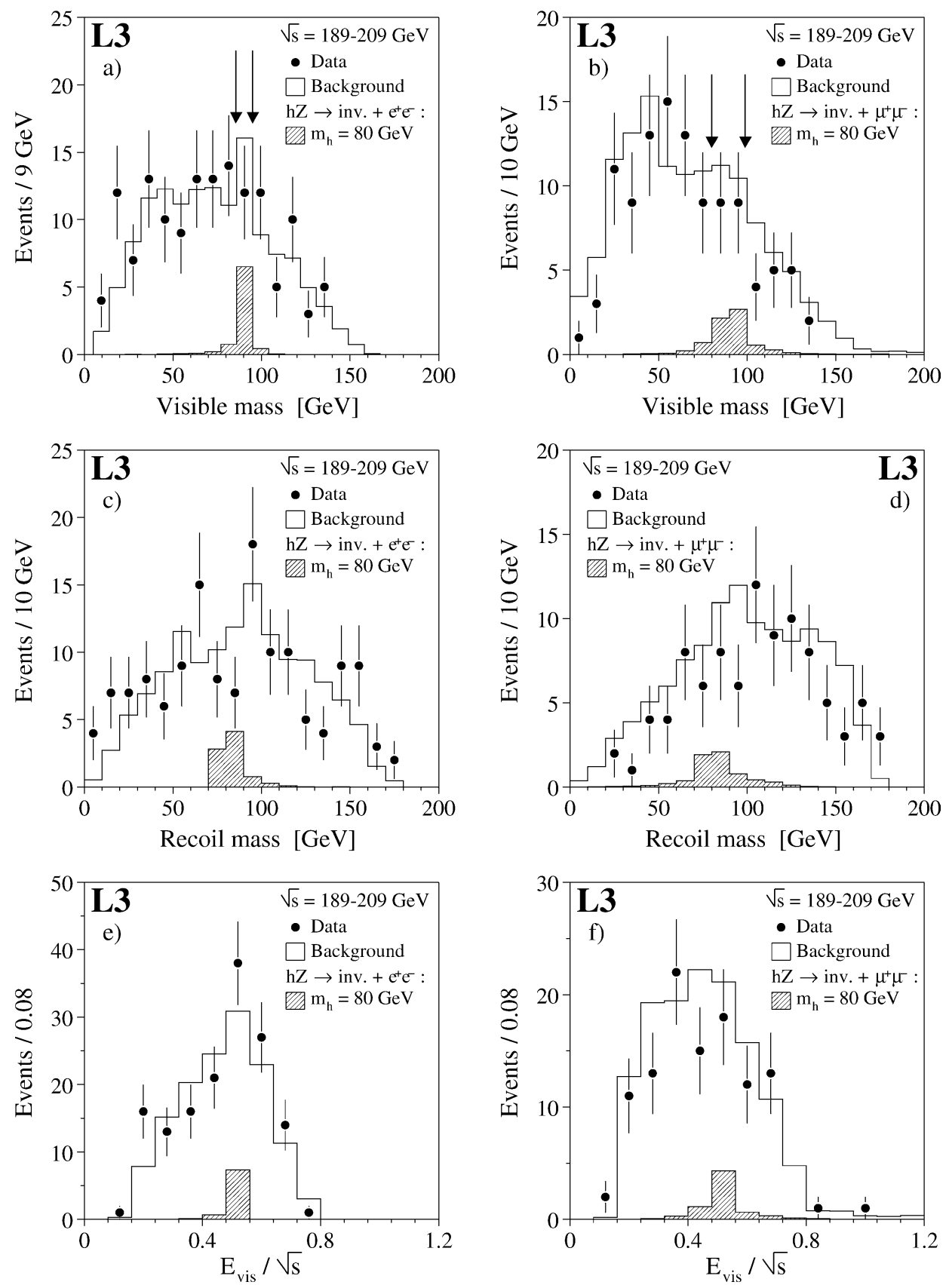

Fig. 3. Distributions of the visible and recoil masses and of the visible energy for events with electrons or muons and missing energy. The dots represent the data, the open area the sum of all background contributions and the hatched histogram the expectation for a signal. The selection criteria on the visible masses are illustrated by the arrows.

consistent with the Standard Model background expectations of 9.7 and 11.1 events, respectively, largely due to four-fermion final states. These results are sum- marised in Table 4 while the signal efficiencies are detailed in Table 3. The distributions of the visible mass of the events, after all other cuts are applied, are 

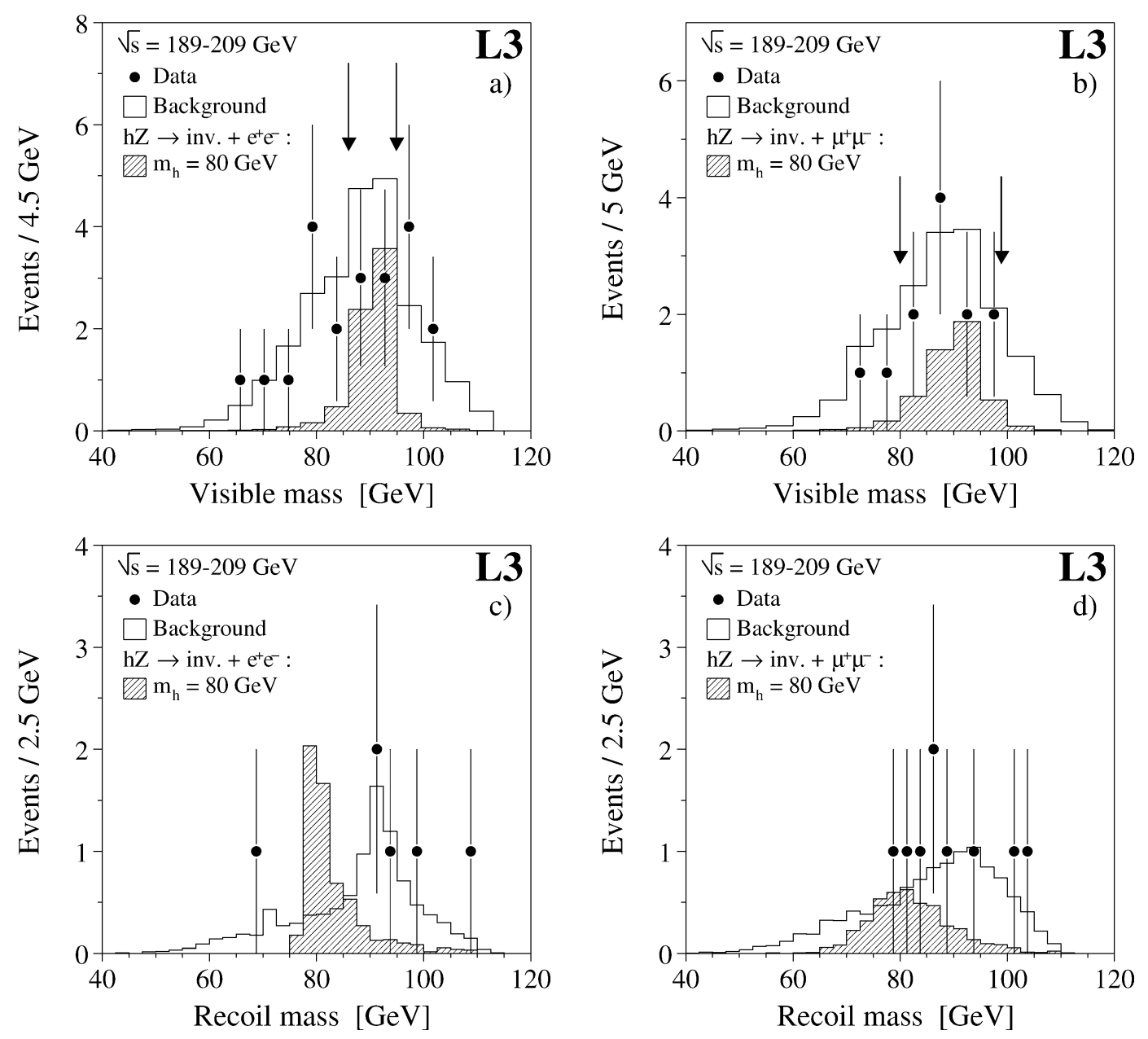

Fig. 4. Distributions of the visible mass of events selected by the analysis of final states with (a) electrons and missing energy and (b) muons and missing energy after. The selection criteria on the visible masses are illustrated by the arrows. Distributions of the recoil mass after the application of all cuts are shown in (c) for electrons and (d) for muons. The dots represent the data, the open area the sum of all background contributions and the hatched histogram the expectation for a signal.

shown in Fig. 4(a) and (b), while Fig. 4(c) and (d) show the distributions of the recoil mass. No indication for a Higgs signal is found in these distributions.

\section{Results}

No evidence is found for a signal due to the production of invisibly-decaying Higgs bosons in association with a $\mathrm{Z}$ boson decaying into hadrons, electrons or muons either in the total counts of events or in the dis- tributions of the discriminant variables and the recoil masses. The results of this search are therefore expressed in terms of limits on $m_{\mathrm{h}}$. In the hypothesis that an invisibly-decaying Higgs boson is produced with the same cross section of the Standard Model Higgs boson, a technique based on a log-likelihood ratio [5] is used to calculate the confidence level $1-\mathrm{CL}_{\mathrm{b}}$ that the observed events are consistent with background expectation. The distributions of the final discriminating variables of the hadron selection, presented in Fig. 2(e) and (f), and of the recoil masses to the lepton system, presented in Fig. 4(c) and (d), are used 

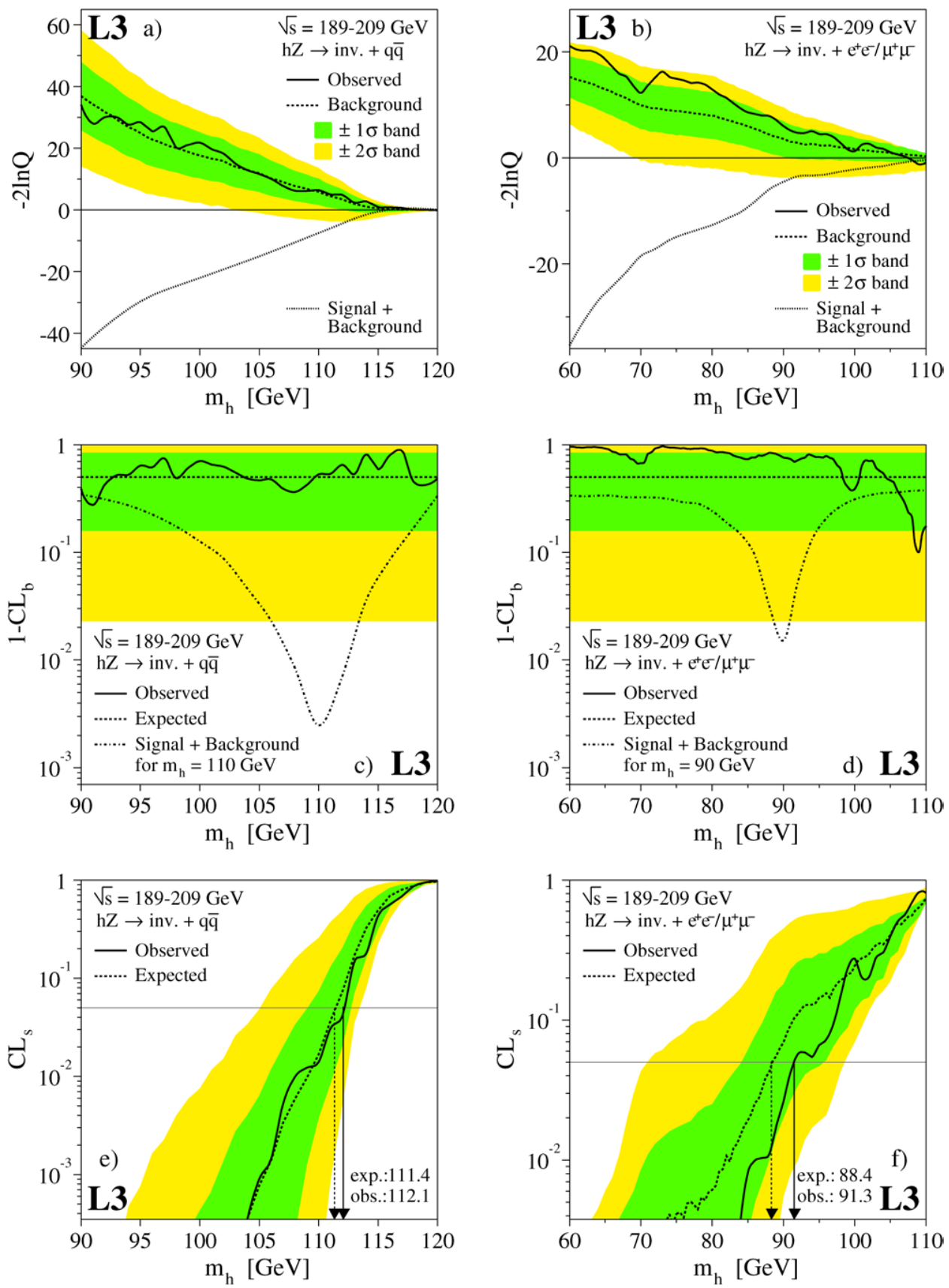

Fig. 5. Distributions as a function of $m_{\mathrm{h}}$ of the log-likelihood ratio for (a) the hadron and (b) the lepton analyses; of the $1-\mathrm{CL}_{\mathrm{b}}$ estimator for (c) the hadron and (d) the lepton analyses; of the $\mathrm{CL}_{\mathrm{S}}$ estimator for (e) the hadron and (f) the lepton analyses, together with the expected and observed lower limits on $m_{\mathrm{h}}$.

in the calculation which yields the results presented in Fig. 5 for the hadron and lepton analyses in terms of the log-likelihood ratio and $1-\mathrm{CL}_{\mathrm{b}}$ as a function of $m_{\mathrm{h}}$. No structure which could hint to the presence of a signal is observed. The confidence level for the presence of the expected signal [5], $\mathrm{CL}_{\mathrm{S}}$, is also de- 
Table 5

Relative systematic uncertainties on the signal efficiency and background normalisation for each analysis channel

\begin{tabular}{|c|c|c|c|c|c|c|}
\hline & \multicolumn{2}{|c|}{$\mathrm{Z} \rightarrow \mathrm{q} \overline{\mathrm{q}}$} & \multicolumn{2}{|c|}{$\mathrm{Z} \rightarrow \mathrm{e}^{+} \mathrm{e}^{-}$} & \multicolumn{2}{|c|}{$\mathrm{Z} \rightarrow \mu^{+} \mu^{-}$} \\
\hline & Signal & Background & Signal & Background & Signal & Background \\
\hline Monte Carlo statistics & $1.4 \%$ & $5.7 \%$ & $1.9 \%$ & $<0.1 \%$ & $2.5 \%$ & $<0.1 \%$ \\
\hline Background cross sections & - & $3.8 \%$ & - & $5.0 \%$ & - & $5.0 \%$ \\
\hline Detector response & $2.0 \%$ & $4.9 \%$ & $2.0 \%$ & $2.7 \%$ & $2.5 \%$ & $4.1 \%$ \\
\hline Total & $2.4 \%$ & $8.4 \%$ & $2.8 \%$ & $5.7 \%$ & $3.5 \%$ & $6.5 \%$ \\
\hline
\end{tabular}
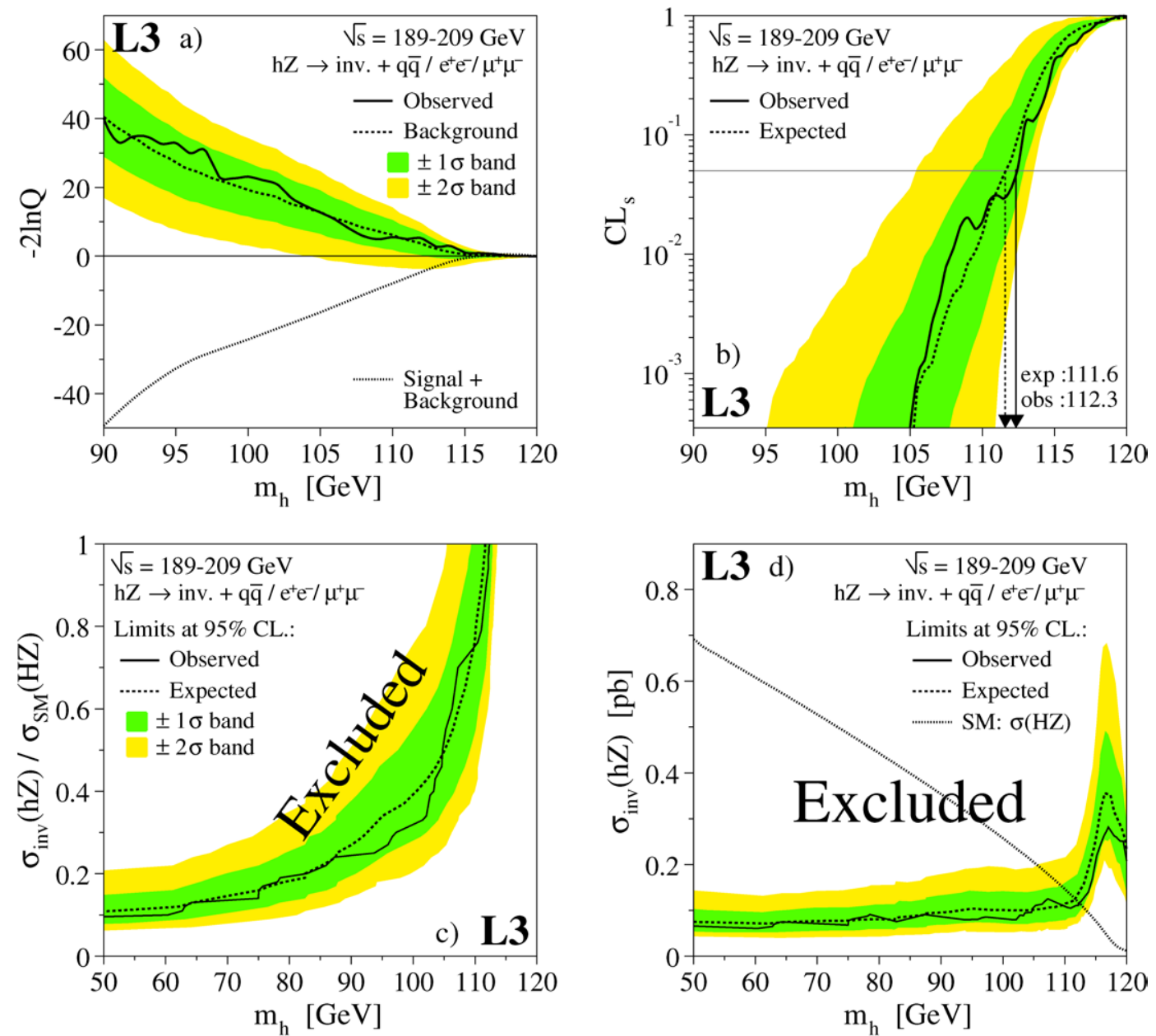

Fig. 6. Distributions as a function of $m_{\mathrm{h}}$ for the combination of the hadron and lepton analyses of (a) the log-likelihood; (b) the CL $\mathrm{L}_{\mathrm{s}}$ estimator with the expected and observed lower limits on $m_{\mathrm{h}}$; (c) upper limits on the ratio of the invisibly-decaying Higgs-boson cross section to the Standard Model one; (d) upper limits on the cross section for the production of an invisibly-decaying Higgs boson.

picted in Fig. 5 for both analyses, as a function of $m_{\mathrm{h}}$. Lower limits at $95 \%$ confidence level (CL) on $m_{\mathrm{h}}$ are derived from the results of the hadron and lep- ton analyses as 112.1 and $91.3 \mathrm{GeV}$, respectively, in good agreement with the expected limits of 111.4 and $88.4 \mathrm{GeV}$. 
These limits include the systematic uncertainties on the signal efficiency and the background normalisation listed in Table 5. These follow from the limited Monte Carlo statistics and from the uncertainties on the cross sections of the background processes. Additional sources of systematic uncertainties, collectively indicated as "detector response" comprise uncertainties in the determination of the energy scale of the detector and possible discrepancies between data and Monte Carlo in the tails of the variables used in the event selection. The inclusion of the systematic uncertainties lowers the limits by about $200 \mathrm{MeV}$.

The results of the combination of the hadron and lepton selections is expressed in terms of the loglikelihood ratio and $\mathrm{CL}_{\mathrm{s}}$ as a function of $m_{\mathrm{h}}$ shown in Fig. 6(a) and (b). A lower limit to the mass of an invisibly-decaying Higgs boson is derived at $95 \% \mathrm{CL}$ as:

$m_{\mathrm{h}}>112.3 \mathrm{GeV}$,

in good agreement with the expected limit of 111.6 GeV. This limit holds in the hypothesis that the invisibly-decaying Higgs boson is produced with the same cross section of the Standard Model Higgs boson. If this hypothesis is relaxed, upper limits as a function of $m_{\mathrm{h}}$ are extracted on the ratio of the invisibly-decaying Higgs-boson cross section to the Standard Model one, as shown in Fig. 6(c). These limits are translated into the upper limits on the cross section for the production of an invisibly-decaying Higgs boson as a function of $m_{\mathrm{h}}$ shown in Fig. 6(d).

\section{References}

[1] S.L. Glashow, Nucl. Phys. 22 (1961) 579; S. Weinberg, Phys. Rev. Lett. 19 (1967) 1264; A. Salam, in: N. Svartholm (Ed.), Elementary Particle Theory, Almqvist \& Wiksell, Stockholm, 1968, p. 367.

[2] P.W. Higgs, Phys. Lett. 12 (1964) 132; P.W. Higgs, Phys. Rev. Lett. 13 (1964) 508; P.W. Higgs, Phys. Rev. 145 (1966) 1156; F. Englert, R. Brout, Phys. Rev. Lett. 13 (1964) 321; G.S. Guralnik, C.R. Hagen, T.W.B. Kibble, Phys. Rev. Lett. 13 (1964) 585.

[3] ALEPH Collaboration, A. Heister, et al., Phys. Lett. B 526 (2002) 191.

[4] DELPHI Collaboration, J. Abdallah, et al., Eur. Phys. J. C 32 (2004) 145; L3 Collaboration, P. Achard, et al., Phys. Lett. B 517 (2001) 319 ;
OPAL Collaboration, G. Abbiendi, et al., Eur. Phys. J. C 26 (2003) 479.

[5] ALEPH, DELPHI, L3 and OPAL Collaborations, The LEP Working Group for Higgs Boson Searches, Phys. Lett. B 565 (2003) 61.

[6] ALEPH Collaboration, H. Heister, et al., Phys. Lett. B 544 (2002) 25;

L3 Collaboration, P. Achard, et al., Phys. Lett. B 583 (2004) 14 ;

OPAL Collaboration, G. Abbiendi, et al., Phys. Lett. B 597 (2004) 11.

[7] L3 Collaboration, P. Achard, et al., Phys. Lett. B 589 (2004) 89.

[8] A. Djouadi, et al., Phys. Lett. B 376 (1996) 220;

K. Griest, H.E. Haber, Phys. Rev. D 37 (1998) 719.

[9] K. Belotsky, et al., Phys. Rev. D 68 (2003) 054027.

[10] S.P. Martin, J.D. Wells, Phys. Rev. D 60 (1999) 035006.

[11] F. de Campos, et al., Phys. Rev. D 55 (1997) 1316, and references therein.

[12] T. Binoth, J.J. van der Bij, Z. Phys. C 75 (1997) 17.

[13] L3 Collaboration, B. Adeva, et al., Nucl. Instrum. Methods A 289 (1990) 35;

L3 Collaboration, O. Adriani, et al., Phys. Rep. 236 (1993) 1; J.A. Bakken, et al., Nucl. Instrum. Methods A 275 (1989) 81;

O. Adriani, et al., Nucl. Instrum. Methods A 302 (1991) 53;

B. Adeva, et al., Nucl. Instrum. Methods A 323 (1992) 109;

K. Deiters, et al., Nucl. Instrum. Methods A 323 (1992) 162; M. Chemarin, et al., Nucl. Instrum. Methods A 349 (1994) 345; M. Acciarri, et al., Nucl. Instrum. Methods A 351 (1994) 300; G. Basti, et al., Nucl. Instrum. Methods A 374 (1996) 293;

A. Adam, et al., Nucl. Instrum. Methods A 383 (1996) 342.

[14] L3 Collaboration, M. Acciarri, et al., Phys. Lett. B 418 (1997) 389.

[15] L3 Collaboration, M. Acciarri, et al., Phys. Lett. B 485 (2000) 85.

[16] DELPHI Collaboration, J. Abdallah, et al., Eur. Phys. J. C 32 (2004) 475; OPAL Collaboration, G. Abbiendi, et al., Eur. Phys. J. C 7 (1999) 407.

[17] PYTHIA versions 5.722 and 6.1 are used; T. Sjöstrand, preprint CERN-TH/7112/93 (1993), revised 1995;

T. Sjöstrand, Comput. Phys. Commun. 82 (1994) 74;

T. Sjöstrand, hep-ph/0001032.

[18] KK2f version 4.13 is used; S. Jadach, B.F.L. Ward, Z. Wạs, Comput. Phys. Commun. 130 (2000) 260.

[19] BHWIDE version 1.03 is used; S. Jadach, W. Placzek, B.F.L. Ward, Phys. Lett. B 390 (1997) 298.

[20] PHOJET version 1.05 is used; R. Engel, Z. Phys. C 66 (1995) 203; R. Engel, J. Ranft, Phys. Rev. D 54 (1996) 4244.

[21] DIAG36 Monte Carlo; F.A. Berends, P.H. Daverfeldt, R. Kleiss, Nucl. Phys. B 253 (1985) 441.

[22] KORALW version 1.33 is used; 
M. Skrzypek, et al., Comput. Phys. Commun. 94 (1996) 216; M. Skrzypek, et al., Phys. Lett. B 372 (1996) 289.

[23] EXCALIBUR version 1.11 is used;

F.A. Berends, R. Kleiss, R. Pittau, Comput. Phys. Commun. 85 (1995) 437.

[24] KandY runs concurrently KORALW version 1.51 and YFSWW3 version 1.16;

S. Jadach, et al., Comput. Phys. Commun. 119 (1999) 272;

S. Jadach, et al., Phys. Rev. D 65 (2002) 090310;

S. Jadach, et al., Comput. Phys. Commun. 140 (2001) 475.

[25] GEANT version 3.15 is used;
R. Brun, et al., Preprint CERN DD/EE/84-1 (1985), revised 1987. The GHEISHA program, H. Fesefeldt, RWTH Aachen Report PITHA 85/02, 1985, is used to simulate hadronic interactions.

[26] S. Catani, et al., Phys. Lett. B 269 (1991) 432;

S. Bethke, et al., Nucl. Phys. B 370 (1992) 310.

[27] L3 Collaboration, P. Achard, et al., Phys. Lett. B 545 (2002) 30.

[28] L3 Collaboration, P. Achard, et al., Phys. Lett. B 600 (2004) 22. 